
M. van Vliet
M. Kliffen
G. P. Krestin
C. F. van Dijke

\section{Soft tissue sarcomas at a glance: clinical, histological, and MR imaging features of malignant extremity soft tissue tumors}

Received: 16 May 2008

Revised: 26 November 2008

Accepted: 2 December 2008

Published online: 6 March 2009

(C) The Author(s) 2009. This article is published with open access at

Springerlink.com

M. van Vliet · G. P. Krestin

Department of Radiology,

Erasmus MC, University Medical

Center Rotterdam,

Rotterdam, The Netherlands

\section{Kliffen}

Department of Pathology,

Maasstad Ziekenhuis,

Rotterdam, The Netherlands

C. F. van Dijke

Department of Radiology,

Medisch Centrum Alkmaar,

Alkmaar, The Netherlands
Present address:

M. van Vliet $(\bowtie)$

Department of Radiology,

Kennemer Gasthuis,

P.O. Box 417, 2000 AK

Haarlem, The Netherlands

e-mail: mvanvliet16@hotmail.com

Tel.: +31-23-5453679

\begin{abstract}
Soft tissue sarcomas comprise approximately $1 \%$ of malignant tumors. There are more than 50 subtypes, but pleomorphic sarcoma, liposarcoma, leiomyosarcoma, synovial sarcoma, and malignant peripheral nerve sheath tumor account for $75 \%$. Differentiation between these subtypes is difficult because they often
\end{abstract}

present with a painless enlarging mass, and share many histological and MR imaging features. Nonetheless, subdifferentiation is important because the different subtypes have different prognoses and therapeutic strategies. In this manuscript we discuss the clinical, histological, and MR imaging features of soft tissue sarcomas according to the WHO classification. An overview is provided and differentiating features are discussed that can help to narrow down the differential diagnosis.

Keywords Soft tissue sarcoma . Magnetic resonance imaging . Histology $\cdot$ WHO

\section{Introduction}

Soft tissue sarcomas (STS) account for approximately $1 \%$ of malignant tumors. There are more than 50 subtypes, but pleomorphic sarcoma (formerly known as malignant fibrous histiocytoma, MFH), liposarcoma, leiomyosarcoma, synovial sarcoma, and malignant peripheral nerve sheath tumor account for $75 \%$. Approximately $75 \%$ arise in the extremities, but the trunk wall, retroperitoneum (RP), and head and neck are also fairly common locations [1]. Soft tissue sarcomas usually do not arise from dedifferentiation of benign soft tissue tumors but develop de novo. The etiology of STS is unknown but some factors are associated with a higher risk, including genetic and environmental factors (e.g., chemical carcinogens), irradiation, viral infections (HHV-8), and immune deficiency. Sporadic cases also occur in areas of scarring and surgical implants [2]. STS usually present as painless soft tissue masses, often large at the time of diagnosis, and they metastasize hematogenously, mainly to the lung. Initial assessment of the lesion should be done with imaging before biopsy as biopsy tracts may produce artifacts, but more importantly because imaging features can be used to choose the optimal biopsy location (e.g., enhancing area of a large tumor) and the optimal biopsy route to prevent spreading of tumor cells through multiple compartments [3]. MRI is the imaging modality of choice since it has the best tissue discrimination and allows multiplanar imaging. 
Conventional radiography can be used to detect bone involvement and calcifications in the tumor [4]. The final diagnosis of STS is made histologically, allowing differentiation between benign and malignant masses and, in case of malignancy, to establish the histological grade and subtype of sarcoma. Treatment of STS requires a multidisciplinary approach. Surgery remains the therapeutic modality of choice, but the extent of surgery required for STS, as well as the role of additional radiotherapy and chemotherapy, either pre- or postoperatively, is still the subject of research and discussion [2].

In 2002 the World Health Organization (WHO) released a new classification of soft tissue tumors [1] to establish a uniform and comprehensive nomenclature for the soft tissue tumor subtypes, with inclusion of the many commonly used synonyms (Table 1). Tumors were divided into nine groups: adipocytic tumors, fibroblastic/myofibroblastic tumors, so-called fibrohistiocytic tumors, smooth muscle tumors, pericytic (perivascular) tumors, skeletal muscle tumors, vascular tumors, chondro-osseous tumors, and tumors of uncertain differentiation. Tumors in these groups were subdivided into four categories according to their biological behavior: benign, intermediate (locally aggressive), intermediate (rarely metastasizing), and malignant. In this review we discuss the imaging characteristics of malignant soft tissue tumors with a focus on tumors occurring in the extremities and provide an overview of differentiating features.

\section{Adipocytic tumors}

\section{Dedifferentiated liposarcoma}

Dedifferentiated liposarcomas are malignant adipocytic tumors showing transition from atypical lipomatous tumor/ well-differentiated liposarcoma (ALT/WDL, intermediate (locally aggressive) tumors according to the WHO classification) to a nonlipogenic sarcoma of variable histological grade. Dedifferentiated areas most frequently resemble unclassified pleomorphic sarcoma, but may exhibit heterologous differentiation (e.g., myogenic, osteo/chondrosarcomatous) [1]. Dedifferentiation can occur in primary lesions but more often in recurrences or metastases. Dedifferentiation is seen in up to $10 \%$ of ALT/WDL, most often in RP tumors [5]. On MRI, ALT/WDL present as predominantly lipomatous tumors with thick septa $(>2 \mathrm{~mm})$, globular and/or nodular areas, and/or associated masses [6]. The nonlipomatous component has low signal intensity (SI) on T1-weighted images (T1WI) and various SIs on T2WI, depending on the type of tissue present. The thick septa or nodules enhance markedly after contrast administration [7-9]. Differentiation between welldifferentiated and dedifferentiated liposarcoma by using MR imaging is difficult. As dedifferentiation occurs within the well-differentiated liposarcoma, imaging characteristics are similar. Dedifferentiation is suggested by the additional presence of a focal nodular nonlipomatous region greater than $1 \mathrm{~cm}$ in size with a low to intermediate SI on T1WI and intermediate to high SI on T2WI [9]. Clinically dedifferentiation is suspected when a tumor suddenly increases in size (Fig. 1).

\section{Myxoid liposarcoma}

Myxoid liposarcomas are the second most common subtype of liposarcoma and they occur mainly in young adults, often in the deep soft tissues of the thigh. Macroscopically myxoid liposarcomas are well-circumscribed, multinodular, intramuscular tumors without prominent tumor necrosis. They may have large mucoid pools creating a "pulmonary edema" growth pattern. Myxoid liposarcomas often recur and metastasize to unusual

Table 1 Synonyms used for soft tissue tumor subtypes

$\begin{array}{ll}\begin{array}{l}\text { Myxoid liposarcoma } \\ \text { Myxofibrosarcoma }\end{array} & \begin{array}{l}\text { Myxoid/round cell liposarcoma, round cell liposarcoma } \\ \text { Myxoid MFH }\end{array} \\ \begin{array}{l}\text { Low grade fibromyxoid sarcoma } \\ \text { UPS }\end{array} & \begin{array}{l}\text { Hyalinizing spindle cell tumor with giant rosettes, fibrosarcoma fibromyxoid type } \\ \text { Embryonal RMS }\end{array} \\ \begin{array}{l}\text { Myosarcoma, malignant rhabdomyoma, rhabdomyosarcoma, rhabdopoietic sarcoma, } \\ \text { rhabdosarcoma, embryonal sarcoma }\end{array} \\ \text { Alveolar RMS } & \begin{array}{l}\text { Rhabdomyoblastoma, rhabdomyopoietic sarcoma, monomorphous round cell rhabdomyosarcoma } \\ \text { Intravascular bronchioloalveolar tumor, angioglomoid tumor, myxoid angioblastomatosis }\end{array} \\ \text { Angithelioid haemangioendothelioma } & \begin{array}{l}\text { Lymphangiosarcoma, hemangiosarcoma, hemangioblastoma, malignant hemangioendothelioma, } \\ \text { malignant angioendothelioma }\end{array} \\ \text { Extraskeletal osteosarcoma } & \text { Soft tissue osteosarcoma } \\ \text { Synovial sarcoma } & \text { Tendosynovial sarcoma, synovial cell sarcoma, malignant synovioma, synovioblastic sarcoma } \\ \text { Clear cell sarcoma } & \text { Malignant melanoma of soft parts } \\ \text { Extraskeletal myxoid chondrosarcoma } & \text { Chordoid sarcoma }\end{array}$




\section{Adipocytic Tumors}

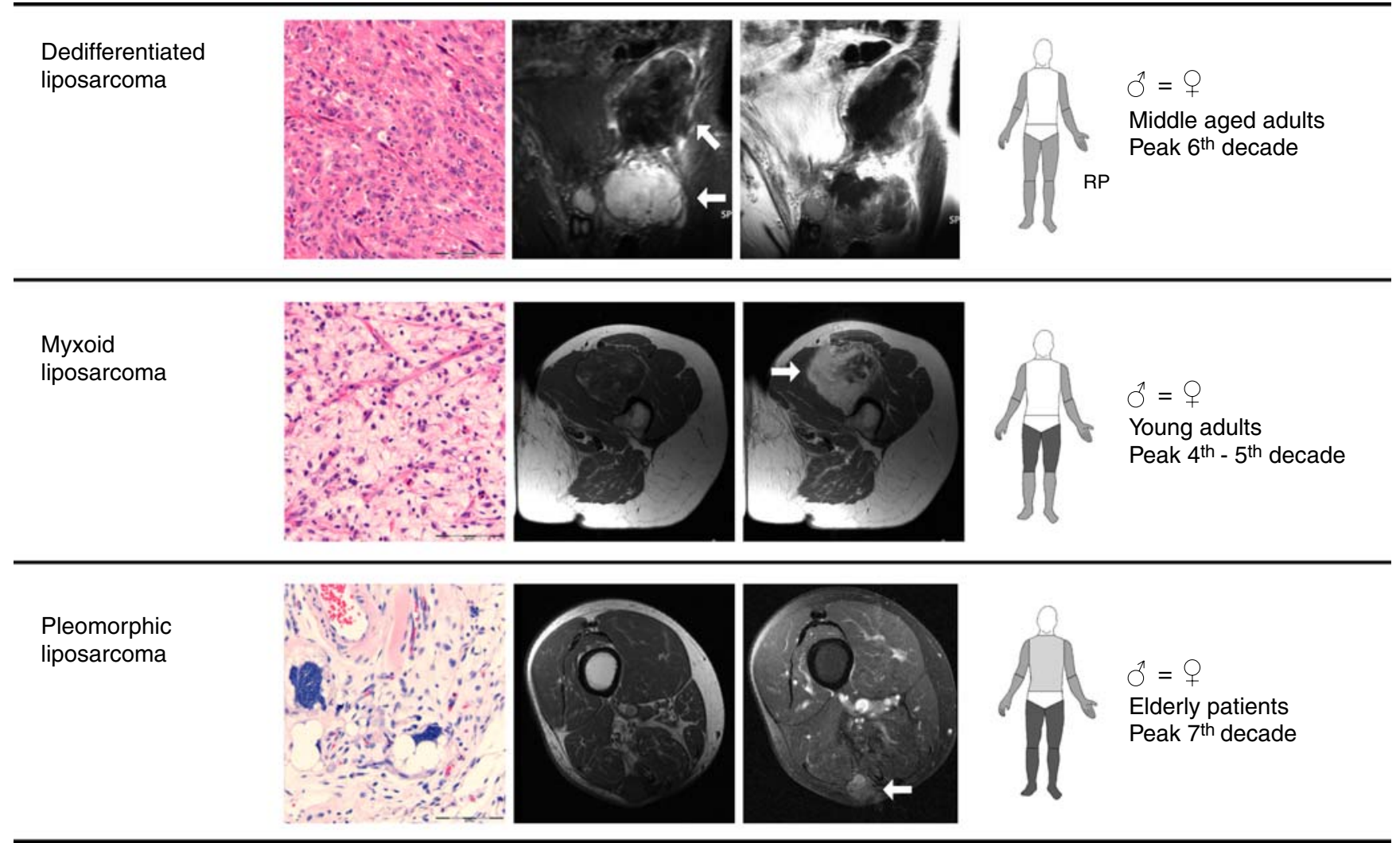

Fig. 1 Left to right Histological image, MR images, most common sites of involvement, male/female ratio, and age distribution of the adipocytic tumors. MRI of dedifferentiated liposarcoma (top row) obtained using coronal T2WI with fat-suppression and T1WI after contrast administration; myxoid liposarcoma (middle row) obtained

locations such as the RP and the opposite extremity, even before spreading to the lungs $[1,5]$. On MRI tumors are characterized by lacy or linear foci of high SI within a low intensity mass on T1WI. The foci represent small quantities of fat within the tumor, usually no more than $10 \%$ of the total volume [10]. Tumors have a high SI on T2WI, due to the myxoid matrix, and most show heterogeneous enhancement with nonenhancing areas of mucinous material and enhancing areas with high vascularity and cellularity $[7-9,11]$. Some present as cystic masses with sharply demarcated margins, which can lead to misdiagnosis as a benign cyst. However, after contrast administration these tumors show a solid area with intense enhancement [12]. The round cell (highly cellular) variant has a nonspecific heterogeneous appearance (Fig. 1).

Pleomorphic liposarcoma

Pleomorphic liposarcomas are the least common subtype of liposarcoma and they occur in elderly patients, mainly in using axial T1WI before and after contrast administration; pleomorphic liposarcoma (bottom row) obtained using axial T1WI before contrast administration and T1WI with fat-suppression after contrast administration. For sites of involvement darker gray indicates higher frequency. $R P$ retroperitoneum

the deep soft tissues of the extremities. Macroscopically pleomorphic liposarcomas can be well circumscribed but nonencapsulated, as well as ill defined and infiltrative and they contain myxoid areas, areas of necrosis and/or hemorrhage, and a variable number of pleomorphic lipoblasts $[1,5]$. On MRI pleomorphic liposarcomas are difficult to differentiate from other aggressive sarcomas because they present as heterogeneous masses (low on T1WI, high on T2WI, with heterogeneous enhancement) and because they contain little or no fat. They usually show well-circumscribed margins [7-9] (Fig. 1).

\section{(Myo)fibroblastic tumors}

Adult fibrosarcoma

Adult fibrosarcoma has become a rare diagnosis as most tumors that were formerly classified as fibrosarcoma are nowadays classified as fibromatosis, pleomorphic sarcoma, malignant peripheral nerve sheath tumor (MPNST), and 
synovial sarcoma based on specific immunohistochemical and/or molecular techniques. Adult fibrosarcoma is no longer considered a separate entity and is therefore not included in this discussion.

\section{Myxofibrosarcoma}

Myxofibrosarcomas are among the most common sarcomas in elderly patients and they mainly occur in the superficial soft tissues of the lower limbs. Histologically myxofibrosarcomas show multinodular growth with incomplete fibrous septa and a myxoid stroma, with a broad spectrum of cellularity, pleomorphism, and proliferative activity [1, 5]. Myxofibrosarcomas resemble myxoid liposarcomas due to their myxoid and multinodular nature. The lesions often show intermediate SI on T1WI and high SI on T2WI with low SI septa [10]. After contrast administration heterogeneous nodular and peripheral contrast enhancement is seen [13]. Cystic formation is possible $[13,14]$. Fat is not present in myxoid MFH so this can be used to differentiate myxofibrosarcomas from myxoid liposarcomas. Wada et al. published a case report of a myxofibrosarcoma with infiltrative growth pattern (no apparent nodular lesion). The tumor spread along the fascial planes and muscles and showed low SI on T1WI and high SI on T2WI without calcifications on plain radiography [15] (Fig. 2).

\section{Low grade fibromyxoid sarcoma}

Low grade fibromyxoid sarcomas occur in the proximal extremities or trunk of young to middle-aged adults. Typically these sarcomas are well-circumscribed masses without hemorrhage or necrosis [5]. Histologically the tumors consist of bland spindle-shaped cells in a variably fibrous and myxoid stroma, alternating in different areas of the tumor $[1,5]$, resulting in slightly inhomogeneous masses with intermediate SI on T1WI and high SI on T2WI. De Schepper et al. described a case of a wellcircumscribed low grade fibromyxoid sarcoma with pe-

\section{(Myo) Fibroblastic Tumors}

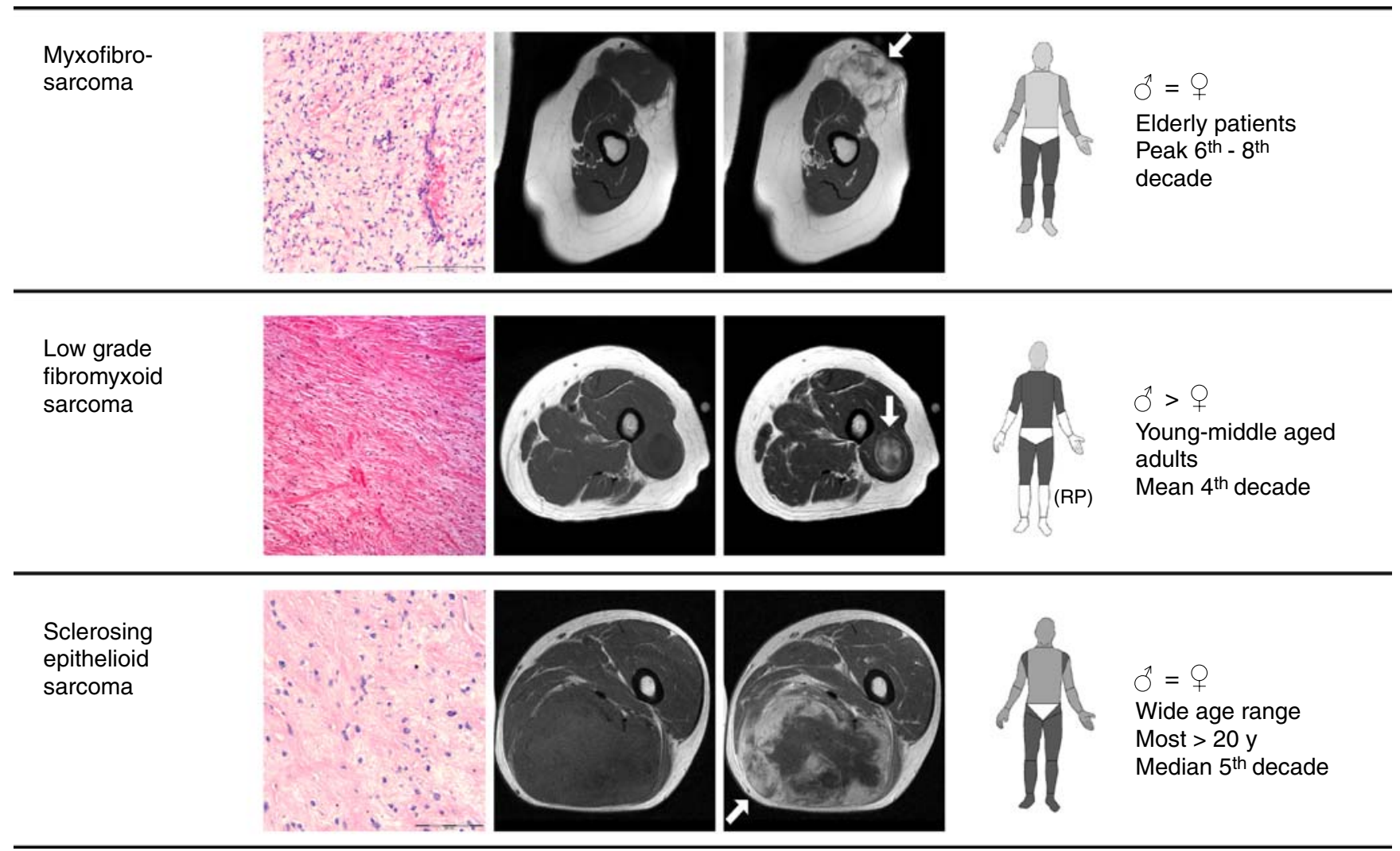

Fig. 2 Left to right Histological image, MR images, most common sites of involvement, male/female ratio, and age distribution of the (myo)fibroblastic tumors. MRI obtained using axial T1WI before and after contrast administration. Low grade fibromyxoid sarcoma (middle row) images courtesy of Dr. C.S.P. van Rijswijk, Leiden University Medical Center, Leiden, the Netherlands. For sites of involvement darker gray indicates higher frequency. $R P$ retroperitoneum 
ripheral contrast enhancement and progressive central filling [10]. Koh et al. described two cases. One was well circumscribed with multiple intralesional nodules, some with a target-like appearance, the other showed invasive features without multinodularity. Both showed heterogeneous contrast enhancement [16] (Fig. 2).

\section{Sclerosing epithelioid fibrosarcoma}

Sclerosing epithelioid fibrosarcomas are rare sarcomas of fibroblastic nature simulating poorly differentiated carcinoma or sclerosing lymphoma. Median age of occurrence is 45 years. The main sites of involvement are the lower extremities and limb girdle. Tumors can contain myxoid, cystic, and calcified areas but necrosis is uncommon $[1,5]$. These tumors can present as a painful mass. Christensen et al. described a case of sclerosing epithelioid fibrosarcoma with a zonal architecture on MRI: a large central core of very low SI and a peripheral rim of intermediate to high SI on both T1WI and T2WI [17]. Despite their wellcircumscribed appearance on MRI, infiltrative margins are commonly found, leading to a local recurrence rate of more than 50\% [1] (Fig. 2).

\section{So-called fibrohystiocytic tumors}

Undifferentiated pleomorphic sarcoma

Undifferentiated pleomorphic sarcomas (UPS) are tumors formerly known as malignant fibrous histiocytoma (MFH). MFH used to be regarded as a distinct tumor type derived from histiocytes representing the most common type of soft tissue sarcoma in adults. However, in recent years this has been abandoned and it is now considered to be a morphologic manifestation of a variety of poorly differentiated neoplasms. UPS are tumors without specific light microscopic features apart from collagen production and UPS is therefore a diagnosis of exclusion. Histologically they are very heterogeneous in appearance and cellularity, but they all contain pleomorphic cells. Some forms have abundant giant cells or a diffuse inflammatory component (giant cell/inflammatory UPS). Undifferentiated pleomorphic sarcomas typically present as painless, circumscribed, multinodular, lobulated tumors with degeneration. The inflammatory variant may present with systemic symptoms, such as fever and weight loss and leukocytosis. Calcification or ossification within the tumor can be seen in $5-20 \%$ of cases, usually curvilinear or punctuate, at the periphery of the lesion. Bone erosion or infiltration is common $[1,5]$. On MRI SI is nonspecific, depending on the cellularity and myxoid content of the lesion and the presence of hemorrhage, necrosis, and/or calcification. Contrast enhancement is often more pronounced in the tumor periphery than in the tumor center because of central location of hemorrhage, necrosis, and myxoid components [13, 14, 18]. Tateishi et al. described 11 pleomorphic/storiform MFHs of the chest wall. Signal characteristics on MRI were high SI on T2WI (all), inhomogeneous enhancement in eight, and homogeneous enhancement in three, all three of which were localized lesions, containing fibrous component and no degeneration [14] (Fig. 3).

\section{Smooth muscle tumors}

\section{Leiomyosarcoma}

Leiomyosarcomas are tumors composed of cells showing distinct smooth muscle features. The most common sites of involvement are the RP and large blood vessels, but leiomyosarcomas also account for approximately $10 \%$ of limb sarcomas. Leiomyosarcomas occur in intramuscular and subcutaneous locations. Borders of the superficial tumors are often better defined than their deep counterparts. Histologically they are usually highly cellular but areas of fibrosis, myxoid change, and necrosis may be present $[1,5]$. Leiomyosarcomas usually present as nonspecific soft tissue masses with low-intermediate SI on T1WI and high SI on T2WI [18]. Leiomyosarcomas are mostly irregular, poorly outlined tumors without a limiting membrane [19]. Large masses usually show central necrosis and a thick, irregular rim-like enhancement after contrast administration on MRI, with bone involvement in $10 \%$ of cases [10] (Fig. 4).

\section{Skeletal muscle tumors}

\section{Embryonal rhabdomyosarcoma}

Embryonal rhabdomyosarcomas (RMS) are primitive tumors with features of embryonic skeletal muscle. Commonly involved sites are head and neck and the genitourinary system; less than $10 \%$ occur in the extremities. RMS usually occur in children under the age of 10 years. Histologically RMS consist of primitive mesenchymal cells in various stages of myogenesis, forming alternating regions of dense cellularity and loose myxoid tissue. Bone destruction is found in approximately $25 \%[1,5]$. In general RMS present as heterogeneous, poorly circumscribed masses with isointense to hyperintense SI compared with muscle on T1WI and high SI on T2WI, with marked heterogeneous contrast enhancement. These MRI characteristics are not specific for RMS and, except for the presence of necrosis, do not differ among subtypes [20, 21]. In embryonal RMS hemorrhage, necrosis, and calcifications are rare, resulting in a more homogeneous 


\section{So-Called Fibrohistiocytic Tumors}

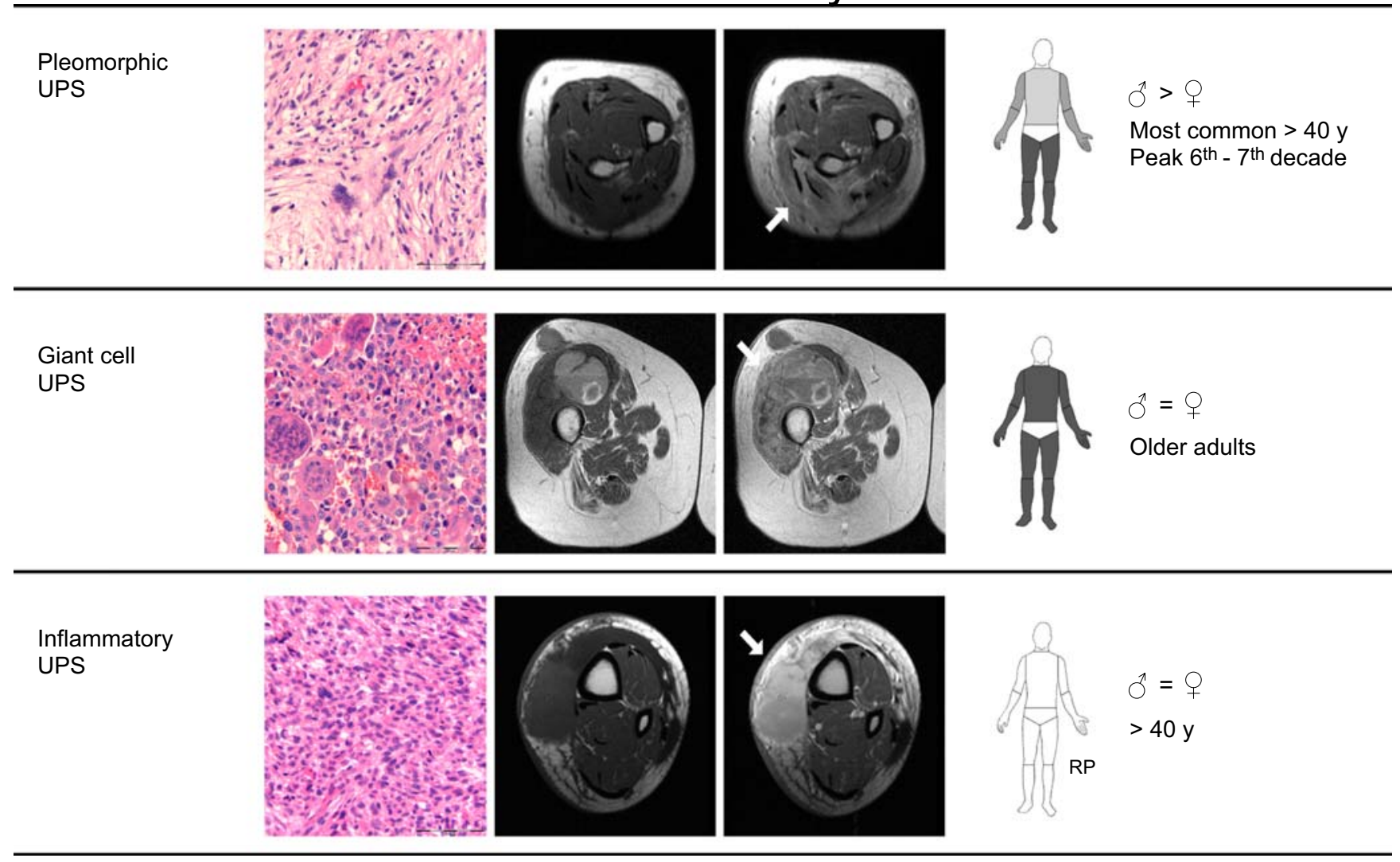

Fig. 3 Left to right Histological image, MR images, most common sites of involvement, male/female ratio, and age distribution of the so-called fibrohistiocytic tumors. MRI obtained using axial T1WI

enhancement pattern. Hagiwara et al. described four rhabdomyosarcomas in the head and neck region with multiple ring enhancing areas resembling bunches of grapes and suggested that this can be characteristic of rhabdomyosarcoma. They called it the "botyroid sign" [21] (Fig. 5). before and after contrast administration. For sites of involvement darker gray indicates higher frequency. UPS undifferentiated pleomorphic sarcoma, $R P$ retroperitoneum

\section{Alveolar rhabdomyosarcoma}

Alveolar rhabdomyosarcomas are primitive, malignant, round cell neoplasms that show partial skeletal muscle differentiation. Alveolar rhabdomyosarcomas commonly arise in the extremities but have no real favored site of

\section{Smooth Muscle Tumors}

Leiomyosarcoma
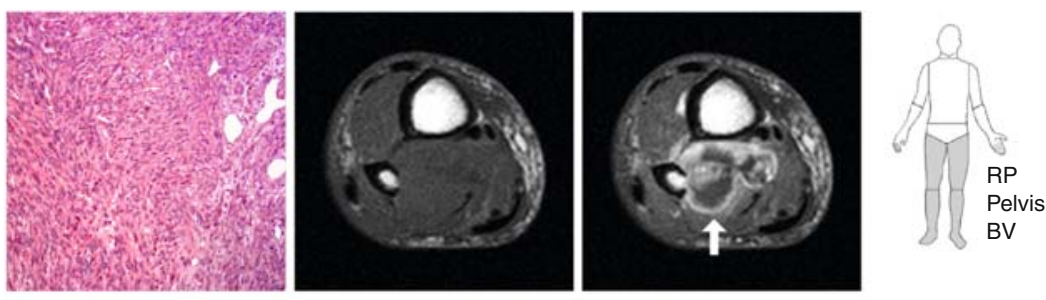

$q>O^{\wedge}(\mathrm{RP}, \mathrm{VCl})$

$\hat{O}=$ Q (other locations)

Middle aged/older adults

Peak $5^{\text {th }-6^{\text {th }}}$ decade
Fig. 4 Left to right Histological image, MR images, most common sites of involvement, male/female ratio, and age distribution of the smooth muscle tumors. MRI obtained using axial T1WI before and after contrast administration. For sites of involvement darker gray indicates higher frequency. $R P$ retroperitoneum, $B V$ blood vessels, $V C I$ vena cava inferior 


\section{Skeletal Muscle Tumors}

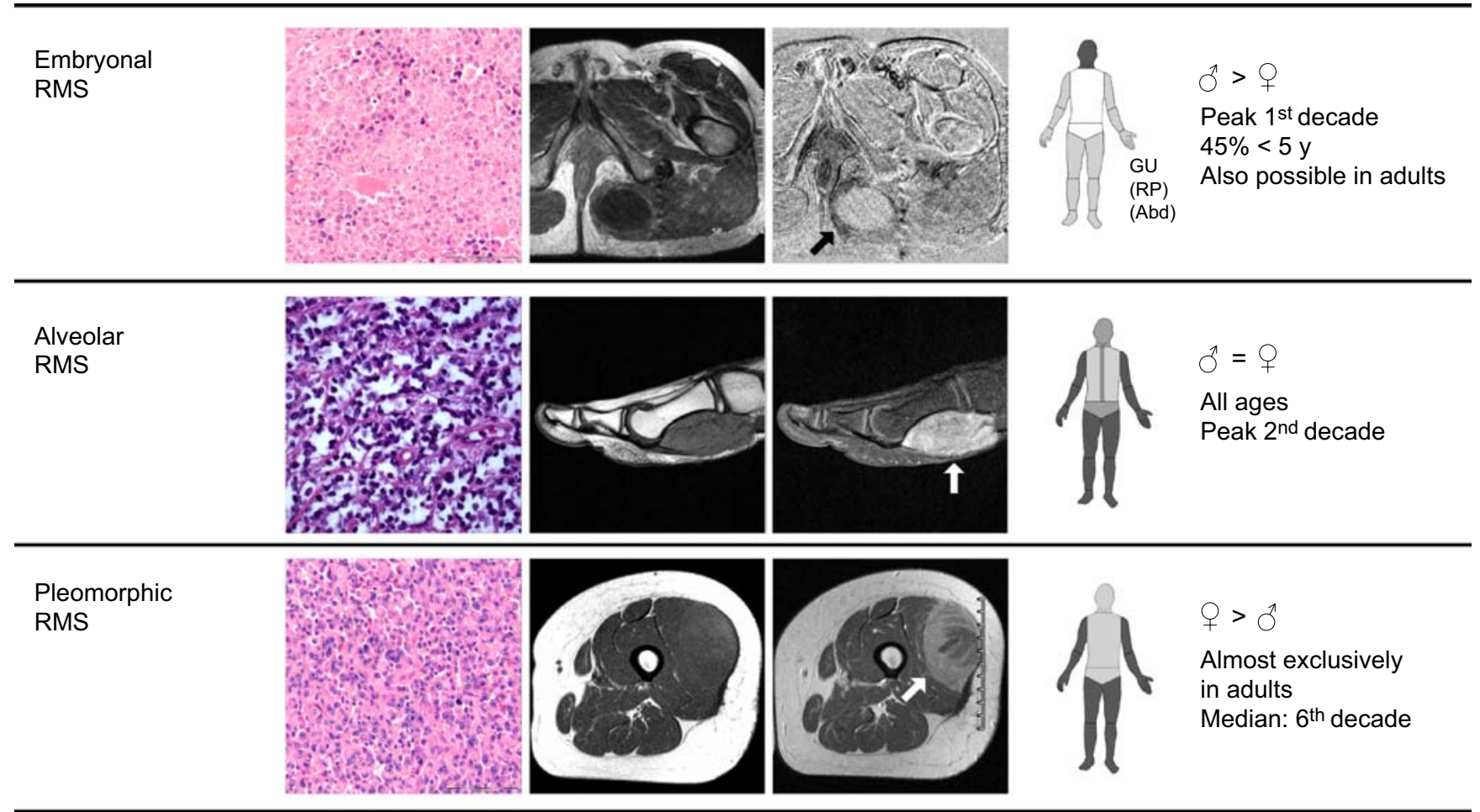

Fig. 5 Left to right Histological image, MR images, most common sites of involvement, male/female ratio, and age distribution of the skeletal muscle tumors. MRI of embryonal RMS (top row) obtained using axial T1WI and T1W subtraction image after contrast administration (dark enhancing); alveolar RMS (middle row) obtained using sagittal T1WI before contrast administration and T1WI with fat-suppression after contrast administration, images

origin. Median age of occurrence is 8 years but they can occur at all ages $[1,5]$. Alveolar rhabdomyosarcomas contain fibrovascular septa that separate the tumor into nests accounting for their alveolar appearance [22]. They present as infiltrative, expansile, soft tissue masses, characterized by multiple areas of necrosis [10]. High flow vessels can be seen, as well as a lobular appearance after contrast administration, but other signal characteristics are nonspecific [20] (Fig. 5).

Pleomorphic rhabdomyosarcoma

Pleomorphic rhabdomyosarcomas are high grade sarcomas consisting of bizarre cells which display evidence of muscle differentiation. In contrast to the other subtypes pleomorphic rhabdomyosarcomas occur almost exclusively in adults, mainly in the deep soft tissues of the extremities. Pleomorphic rhabdomyosarcomas contain variable amounts of hemorrhage and necrosis $[1,5]$. Signal characteristics are nonspecific: isointense to skeletal muscle on T1WI and heterogeneous SI on T2WI. Necrotic foci courtesy of Dr. R.R. van Rijn, Academic Medical Center Amsterdam, the Netherlands; pleomorphic RMS (bottom row) obtained using axial T1WI before and after contrast administration. For sites of involvement darker gray indicates higher frequency. $R M S$ rhabdomyosarcoma, $G U$ genitourinary, $R P$ retroperitoneum, $A b d$ abdominal

are readily identifiable in many cases [1]. Pleomorphic rhabdomyosarcomas have been shown to present with areas of necrosis alternating with areas of marked enhancement and areas of ring-like enhancement around areas of low SI [10] (Fig. 5).

\section{Vascular tumors}

Epithelioid hemangioendothelioma

Epithelioid hemangioendotheliomas (EHE) are rare vascular tumors, occurring at nearly all ages except in early childhood. Most originate from a small vessel, usually a small vein. EHE classically present as fusiform intravascular masses which may resemble a thrombus, expanding the originating vessel without disturbing its architecture. EHE lumens are filled with necrotic debris and dense collagen. EHE are solitary tumors, occurring mainly in either the superficial or deep soft tissues of the extremities and often present as painful nodules, sometimes associated with edema and thromboflebitis $[1,5]$. MRI characteristics of hemangioendothelioma 


\section{Vascular Tumors}

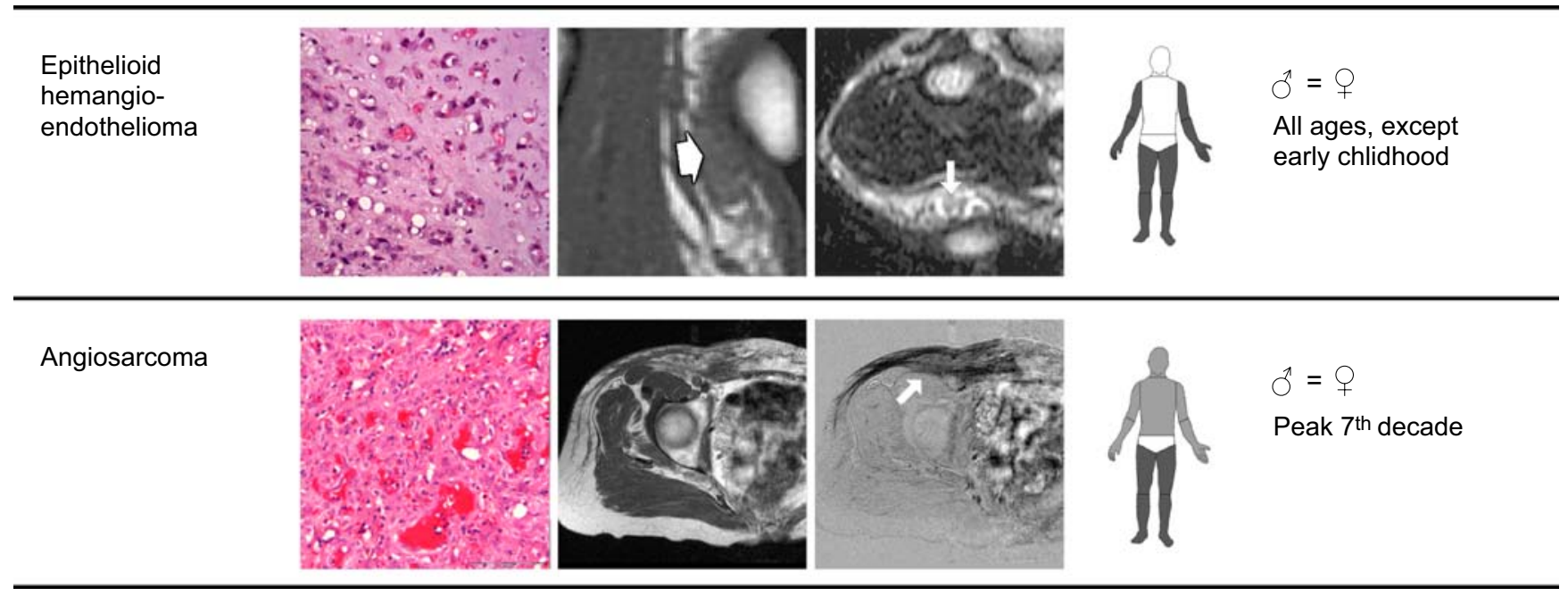

Fig. 6 Left to right Histological image, MR images, most common sites of involvement, male/female ratio, and age distribution of the vascular tumors. MRI of epithelioid hemangioendothelioma (top row) obtained using coronal T1WI before contrast administration and axial T2WI, images courtesy of Dr. J.C. Vilanova et al., Clinica
Girona, Girona, Spain; angiosarcoma (bottom row) obtained using axial T1WI before contrast administration and T1W subtraction image after contrast administration (dark enhancing). For sites of involvement darker gray indicates higher frequency and angiosarcoma are nonspecific [23]. Prominent serpentine vessels may be present. SI of the vascular channels is flowdependent. Sometimes areas with high SI on T1WI can be seen, corresponding to hemorrhage. The presence of necrosis in the tumor is indicative of malignancy [10] (Fig. 6).

\section{Angiosarcoma of soft tissue}

Soft tissue angiosarcomas (AS) are malignant tumors containing cells with morphologic and functional features of endothelial cells. Most AS are cutaneous tumors, less than $25 \%$ present as deep soft tissue masses. AS generally contain both epithelioid and spindle cell areas, forming rudimentary infiltrating vascular channels. Hemorrhage is common and often extensive. AS present as enlarging masses with or without systemic symptoms such as coagulopathy, anemia, hematomas, and bruisability. Associations are described with several conditions: (M)PNST, NF-1, implanted foreign materials, Klippel-Trenaunay, and previous irradiation $[1,5]$. Angiosarcomas show lowintermediate SI on T1WI and relatively high and low signal components on T2WI with marked heterogeneous contrast enhancement. SI on T2WI may reflect the relative proportion of vascular space, cellularity, and stroma in the tumor [24-26] (Fig. 6).

\section{Chondro-Osseous tumors}

Extraskeletal
osteosarcoma

Fig. 7 Left to right Histological image, MR images, most common sites of involvement, male/female ratio, and age distribution of the chondro-osseous tumors. MRI obtained using axial T1WI before and after contrast administration. For sites of involvement darker gray indicates higher frequency 


\section{Chondro-osseous tumors}

\section{Extraskeletal osteosarcoma}

Extraskeletal osteosarcomas (EO) are malignant mesenchymal tumors composed of neoplastic cells that resemble osteoblasts and synthesize bone. All contain bone but they may also have cartilaginous and fibroblastic components. No other lines of differentiation are evident. The most common site of involvement is the thigh. EO are large, deep-seated soft tissue masses with a variable amount of mineralization. EO do not arise from bone but may secondarily involve periosteum, cortex, or the medullary canal. Most develop de novo but associations have been found with previous irradiation and trauma $[1,5]$. On MRI EO often present as well-circumscribed inhomogeneous masses on T1WI and T2WI with inhomogeneous contrast enhancement. Calcification is present in up to $70 \%$ of lesions (presenting as signal voids on all sequences), usually with a large amount of calcific and/or osteoid material. In contrast to myositis ossificans mineralization occurs mainly in the center of the lesion. Fluid-fluid levels are often present. Necrosis, hemorrhage, and cystic change are common. Tumors are often pseudoencapsulated [10, 27, 28] (Fig. 7).

\section{Tumors of uncertain differentiation}

\section{Synovial sarcoma}

Synovial sarcomas are mesenchymal spindle cell tumors which display variable epithelial differentiation, including glandular formation. Histologically there are two types: biphasic and monophasic. The biphasic variant has epithelial and spindle cell components. The monophasic variant contains only spindle cells, often with a hemangiopericytomatous vascular pattern. SS is unrelated to synovium: less than $5 \%$ of SS originate in a joint or bursa. The main sites of involvement are the deep soft tissues of the extremities, especially around the knee and adjacent to joints or tendon sheaths $[1,5]$. Synovial sarcomas are multilobular, septated masses that show heterogeneous SI on T1WI and T2WI and heterogeneous contrast enhancement. Heterogeneity is caused by areas of necrosis, calcification, cysts, and hemorrhage within the tumor $[10,11]$. Triple SI on T2WI (SI is hypo-, iso-, and hyperintense relative to fat) is not specific but suggestive of the diagnosis [29, 30]. Calcification is seen in a third of cases. Focal calcification or ossification ranging from fine stippling to marked calcifications in the periphery of the lesion can be found [18, 31]. Fluid-fluid levels can be present. Smaller lesions can be well-circumscribed and homogeneous and can be mistaken for a benign cystic mass [32]. Up to $20 \%$ show erosion or invasion of adjacent bone [33] (Fig. 8).
Clear cell sarcoma of soft tissue

Clear cell sarcomas of soft tissue are tumors with melanocytic differentiation, typically involving tendons and aponeuroses. The principal sites of involvement are the extremities, mainly the foot and ankle. These tumors occur mainly in young adults. Pain/tenderness is a symptom in about $50 \%$. Histologically clear cell sarcomas of soft tissue present with a uniform, nested, or fascicular growth pattern with thin fibrous septa delineating the tumor cell nests. Melanin and/or giant cells are present in about $50 \%$ of cases. Differentiation from malignant melanoma is impossible on morphological grounds [1,5]. On MRI clear cell sarcomas usually present as well-circumscribed lesions with variable SI on T1WI and T2WI. About $50 \%$ of tumors are slightly hyperintense to muscle on T1WI, probably due to the presence of melanin, and markedly hyperintense to muscle on T2WI [34]. Hourani et al. suggested that clear cell sarcoma should be suspected in a lesion which displays high SI on all sequences, including fat-sat techniques [35]. As clear cell sarcomas are often well-defined and homogeneous and rarely show bone destruction or necrosis, they are often misdiagnosed as benign lesions [10, 31, 33-36] (Fig. 8).

\section{Epithelioid sarcoma}

Epithelioid sarcomas are tumors of unknown lineage with predominantly epithelioid cytomorphology. They may be misdiagnosed as benign granulomatous processes such as rheumatoid nodules. Macroscopically these tumors are small ill-defined dermal or subcutaneous nodules or larger deeper masses involving tendons or fascia. The center of these sarcomas is usually necrotic or hemorrhagic. The main sites of involvement are the flexor surfaces of the hand and forearm. Epithelioid sarcomas occur mainly in young adults. Epithelioid sarcomas are reported to be homogeneous or heterogeneous depending on their location (superficial: homogeneous, deep: heterogeneous) and they are mostly isointense to muscle on T1WI and hyperintense on T2WI with strong enhancement [10, 33]. Chao et al. described a case of epitheloid sarcoma of the thigh presenting as a subcutaneous multinodular mass with a central area of hypointensity and a peripheral portion with serpiginous hyperintensity. Calcifications were present but not visible on MRI [37]. Zevallow-Gieampietri et al. described two cases of epithelioid sarcoma in the perineum. The first tumor was a well-circumscribed mass with homogeneous enhancement. The second tumor was a nonencapsulated tumor, showing heterogeneous enhancement involving subcutaneous tissue and muscle tissue. Neither tumor showed calcifications or bone or skin involvement [38] (Fig. 8). 
Alveolar soft part sarcoma

Alveolar soft part sarcomas (ASPS) are tumors with a high vascularity causing pulsations or bruits over the tumor. The main sites of involvement are the extremities. ASPS mainly occur in young adults. Histologically, tumor cells grow in an organoid or nesting pattern $[1,5]$. Nests are separated by connective tissue containing sinusoidal vascular channels

\section{Tumors of Uncertain Differentiation}

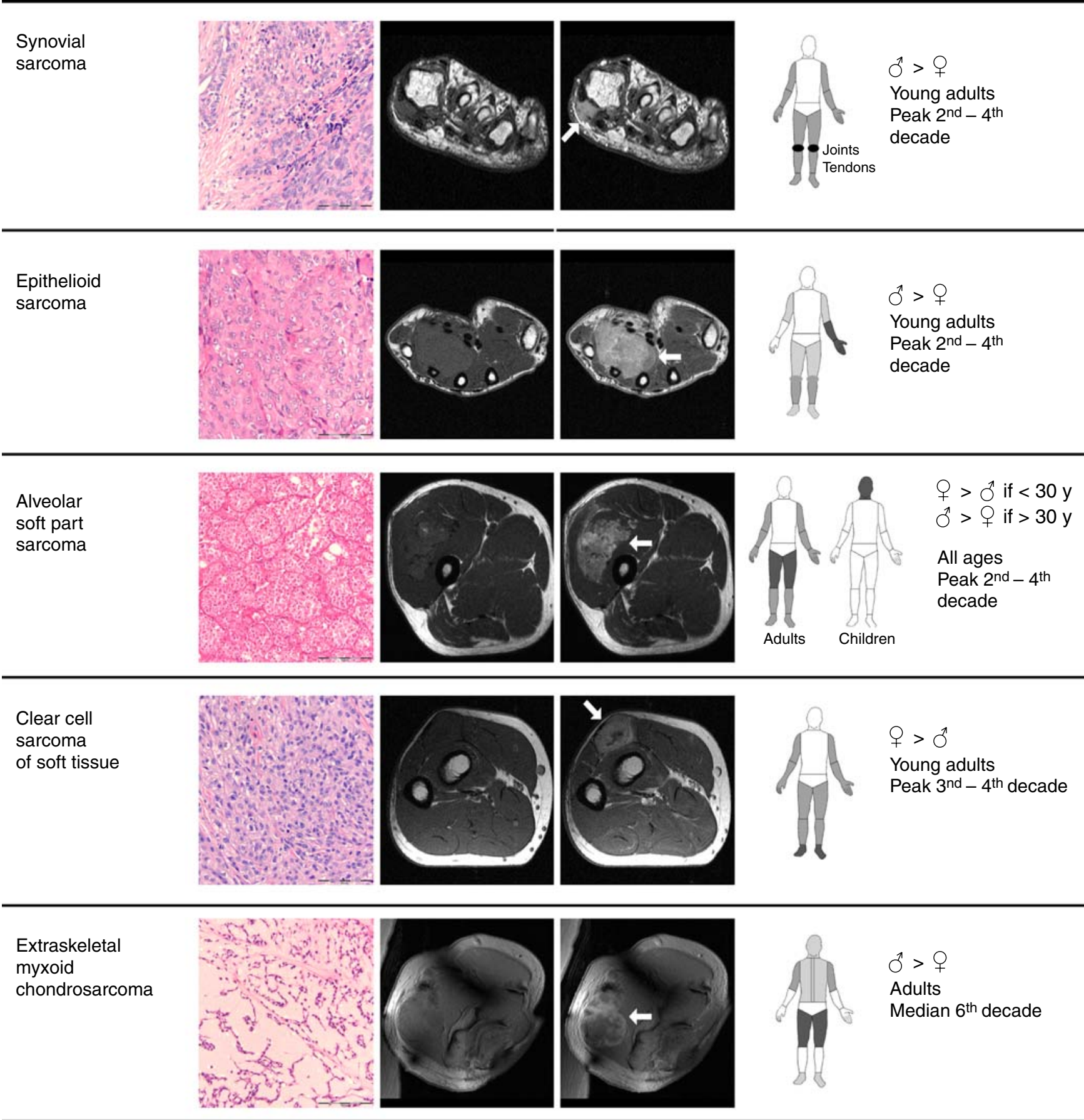

Fig. 8 Left to right Histological image, MR images, most common sites of involvement, male/female ratio, and age distribution of the tumors of uncertain differentiation. MRI obtained using axial T1WI before and after contrast administration. For sites of involvement darker gray indicates higher frequency 
resulting in hypervascular lesions with prominent draining veins on angiography or MRA [39]. ASPS are usually heterogeneous, well-circumscribed lesions with high SI on T1WI and T2WI and intense inhomogeneous contrast enhancement [10]. High SI on T1WI with numerous signal voids is very suggestive of ASPS, the signal voids suggesting rapid flow within distended vessels; however, not all tumors show these signal characteristics. Arteriovenous malformations (AVM) show similar characteristics, but in ASPS an accompanying soft tissue mass is present [40]. Bone erosion may be found but is uncommon [33] (Fig. 8).

\section{Extraskeletal myxoid chondrosarcoma}

Extraskeletal myxoid chondrosarcomas (EMC) are multinodular tumors with abundant myxoid matrix and malignant chondroblast-like cells arranged in networks. Despite the name there is no convincing evidence of cartilaginous differentiation. Main sites of involvement are the deep soft tissues of the proximal extremities. Tumors usually do not contain calcification or bone formation and do not involve adjacent bone. EMC appear as a lobulated soft tissue mass and are usually inhomogeneous and ill-defined, but well delineated and homogeneous variants have been described. On T1WI SI is variable, from equal to muscle to equal to fat. On T2WI SI is as high as or higher than fat. Highly myxoid tumors may have a homogeneous very high SI on T2WI, resembling a cyst or myxoma. Tumors with necrosis or hemorrhage show a more heterogeneous signal $[1,10]$ (Fig. 8).

\section{Differentiating features}

Differentiating between the subtypes of sarcoma is difficult, partly because soft tissue sarcomas are rare tumors, making it difficult to obtain a large series of each subtype, but also because the subtypes are very similar in appearance. The sarcomas are mostly large, deep-seated

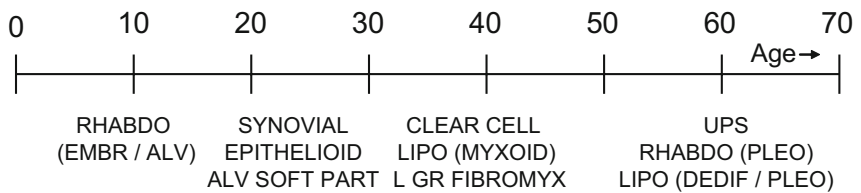

Fig. 9 Age distribution of some of the subtypes of soft tissue sarcoma

tumors, often with hemorrhage and necrosis, making them inherently inhomogeneous masses. There are, however, factors which can help to narrow down the number of possibilities. Important differentiating factors are age and location, the presence or absence of pain, as well as (MR) imaging characteristics (Table 2).

Age

Embryonal and alveolar rhabdomyosarcomas mainly occur in children; sarcomas of uncertain differentiation in young adults; and UPS, liposarcoma, leiomyosarcoma, and pleomorphic rhabdomyosarcomas in older adults (Fig. 9).

\section{Location}

Tumor location (deep/superficial, site of involvement) is also an important differentiating factor. Most tumors are deep seated, but some subtypes, such as myxofibrosarcomas, epithelioid sarcomas, and angiosarcomas, are commonly superficially located. Although most soft tissue sarcomas occur in the proximal lower extremity, some are more common in other locations. Clear cell sarcoma, for example, most commonly occurs in the foot, epithelioid sarcoma in the flexor surface of the arm, and synovial sarcoma near (but not in) joints. Clear cell sarcoma, synovial sarcoma, and epithelioid sarcoma are often associated with tendons/aponeuroses.

Table 2 Differentiating features between sarcoma subtypes

\begin{tabular}{ll}
\hline Calcification & $\begin{array}{c}\text { Well-differentiated liposarcoma, undifferentiated pleomorphic sarcoma, extraskeletal osteosarcoma, } \\
\text { synovial sarcoma } \\
\text { No/little necrosis }\end{array}$ \\
$\begin{array}{l}\text { Myxoid liposarcoma, low grade fibromyxoid sarcoma, sclerosing epithelioid fibrosarcoma, embryonal } \\
\text { rhabdomyosarcoma, clear cell sarcoma } \\
\text { Pain }\end{array}$ & $\begin{array}{l}\text { Sclerosing epithelioid fibrosarcoma, pleomorphic rhabdomyosarcoma, epithelioid hemangioendothelioma, } \\
\text { large angiosarcoma, extraskeletal osteosarcomas, synovial sarcoma, clear cell sarcoma }\end{array}$ \\
Superficial & Myxofibrosarcoma, angiosarcoma, epithelioid sarcoma, leiomyosarcoma, epithelioid hemangioendothelioma \\
Ill-defined & Alveolar rhabdomyosarcoma, pleomorphic rhabdomyosarcoma, epithelioid sarcoma, alveolar soft part sarcoma \\
Bone destruction & Undifferentiated pleomorphic sarcoma, leiomyosarcoma, rhabdomyosarcoma, synovial sarcoma \\
Associated with tendons & Clear cell sarcoma, synovial sarcoma, epithelioid sarcoma \\
Lymph node metastases & Epithelioid sarcoma, synovial sarcoma, rhabdomyosarcoma, clear cell sarcoma, angiosarcoma \\
\hline
\end{tabular}




\section{Pain}

Whereas most sarcomas are painless masses, pain is commonly found in sclerosing epithelioid fibrosarcoma, pleomorphic rhabdomyosarcoma, epithelioid hemangioendothelioma, large angiosarcoma, extraskeletal osteosarcoma, synovial sarcoma, and clear cell sarcoma.

\section{Lymph node metastases}

Most soft tissue sarcomas metastasize hematogenously, mainly to the lung. A few subtypes of sarcoma are known to show lymph node metastases: epithelioid sarcoma, synovial sarcoma, rhabdomyosarcoma, clear cell sarcoma, and angiosarcoma [2].

\section{MR imaging features}

Although the role of MRI in evaluating the extent of sarcomas has already been established, its ability in differentiating between benign and malignant lesions and between the various subtypes of sarcoma is still controversial as most soft tissue tumors have nonspecific imaging findings: intermediate SI on T1WI, high SI on T2WI, and heterogeneous enhancement after contrast administration, due to the presence of hemorrhage and necrosis in the tumor [41]. MR imaging features can, however, be of help in the differential diagnosis if lesions have an atypical appearance, e.g., high SI on T1WI and/or low SI on T2WI. Fat, blood products, and proteinacious fluids have a high SI on T1WI. Therefore dedifferentiated liposarcomas are mostly bright on $\mathrm{T} 1$, and myxoid liposarcoma may show areas of high SI within a low SI mass. High SI on T1WI is also a characteristic feature of alveolar soft part sarcoma. Fibrous tissue, deoxyhemoglobin, hemosiderin, calcium, and air have a low SI on T2WI. Tumors containing fibrous tissue, such as low grade fibrosarcomas and UPS therefore present with areas of low SI on T2WI. Clear cell sarcomas may show both hyperintense areas on T1WI and hypointense areas on T2WI because of the presence of melanin in the tumor (melanin causes shortening of $\mathrm{T} 1$ and $\mathrm{T} 2$ relaxation times). Calcifications are also a differentiating feature: they can be seen in among others well-differentiated liposarcoma, undifferentiated pleomorphic sarcoma, extraskeletal osteosarcoma, and synovial sarcoma. Necrosis is common in most soft tissue sarcomas except in myxoid liposarcomas, low grade fibromyxoid sarcoma, sclerosing epithelioid fibrosarcoma, embryonal rhabdomyosarcoma, and clear cell sarcoma.

In conclusion, although MR characteristics alone are often insufficient to differentiate between the subgroups of soft tissue sarcomas, MRI can limit the number of possibilities and provide important information regarding the optimal biopsy site and tumor extent. Combination of clinical, histological, and imaging characteristics remains essential in the diagnosis and treatment of soft tissue sarcomas.

Open Access This article is distributed under the terms of the Creative Commons Attribution Noncommercial License which permits any noncommercial use, distribution, and reproduction in any medium, provided the original author(s) and source are credited.

\section{References}

1. Fletcher CDM, Unni KK, Mertens FE (eds) (2002) World Health Organization classification of tumors. Pathology and genetics of tumours of soft tissue and bone. IARC, Lyon

2. Cormier JN, Pollock RE (2004) Soft tissue sarcomas. CA Cancer J Clin 54:94-109

3. Varma DG (1999) Optimal radiologic imaging of soft tissue sarcomas. Semin Surg Oncol 17:2-10

4. Massengill AD, Seeger LL, Eckardt JJ (1995) The role of plain radiography, computed tomography, and magnetic resonance imaging in sarcoma evaluation. Hematol Oncol Clin North Am 9:571-604

5. Weiss SW, Goldblum JR (eds) (2001) Enzinger and Weiss's soft tissue tumors. Mosby, St Louis
6. Kransdorf MJ, Bancroft LW, Peterson JJ, Murphey MD, Foster WC, Temple HT (2002) Imaging of fatty tumors: distinction of lipoma and welldifferentiated liposarcoma. Radiology 224:99-104

7. Drevelegas A, Pilavaki M, Chourmouzi D (2004) Lipomatous tumors of soft tissue: MR appearance with histological correlation. Eur J Radiol 50:257-267

8. Barile A, Zugaro L, Catalucci A et al (2002) Soft tissue liposarcoma: histological subtypes, MRI and CT findings. Radiol Med (Torino) 104:140-149

9. Murphey MD, Arcara LK, FanburgSmith J (2005) From the archives of the AFIP: imaging of musculoskeletal liposarcoma with radiologic-pathologic correlation. Radiographics 25:13711395

10. De Schepper AM (ed) (2001) Imaging of soft tissue tumors, 2nd edn. Springer-Verlag, Berlin
11. Laor T (2004) MR imaging of soft tissue tumors and tumor-like lesions. Pediatr Radiol 34:24-37

12. Sung MS, Kang HS, Suh JS et al (2000) Myxoid liposarcoma: appearance at MR imaging with histologic correlation. Radiographics 20:10071019

13. Munk PL, Sallomi DF, Janzen DL et al (1998) Malignant fibrous histiocytoma of soft tissue imaging with emphasis on MRI. J Comput Assist Tomogr 22:819826

14. Tateishi U, Kusumoto M, Hasegawa T, Yokoyama R, Moriyama N (2002) Primary malignant fibrous histiocytoma of the chest wall: CT and MR appearance. J Comput Assist Tomogr 26:558563 
15. Wada T, Hasegawa T, Nagoya $S$, Kawaguchi S, Kaya M, Ishii S (2000) Myxofibrosarcoma with an infiltrative growth pattern: a case report. Jpn J Clin Oncol 30:458-462

16. Koh SH, Choe HS, Lee IJ, Park HR, Bae SH (2005) Low-grade fibromyxoid sarcoma: ultrasound and magnetic resonance findings in two cases. Skeletal Radiol 34:550-554

17. Christensen DR, Ramsamooj R, Gilbert TJ (1997) Sclerosing epithelioid fibrosarcoma: short T2 on MR imaging. Skeletal Radiol 26:619-621

18. Rechl H, Hof N, Gerdesmeier L (2001) (Differential diagnosis of bone and soft tissue tumors by MRI) Differentialdiagnostik von Knochen- und Weichteiltumoren in der MRT. Orthopade 30:528-539

19. Mankin HJ, Casas-Ganem J, Kim JI, Gebhardt MC, Hornicek FJ, Zeegen EN (2004) Leiomyosarcoma of somatic soft tissues. Clin Orthop Relat Res:225-231

20. Kim EE, Valenzuela RF, Kumar AJ, Raney RB, Eftekari F (2000) Imaging and clinical spectrum of rhabdomyosarcoma in children. Clin Imaging 24:257-262

21. Hagiwara A, Inoue Y, Nakayama T et al (2001) The "botryoid sign": a characteristic feature of rhabdomyosarcomas in the head and neck. Neuroradiology 43:331-335

22. Van Rijn RR, Wilde JC, Bras J, Oldenburger F, McHugh KM, Merks JH (2008) Imaging findings in noncraniofacial childhood rhabdomyosarcoma. Pediatr Radiol 38:617-634

23. Vilanova JC, Barcelo J, Smirniotopoulos JG et al (2004) Hemangioma from head to toe: MR imaging with pathologic correlation. Radiographics 24:367-385
24. Nakazono T, Kudo S, Matsuo Y et al (2000) Angiosarcoma associated with chronic lymphedema (Stewart-Treves syndrome) of the leg: MR imaging. Skeletal Radiol 29:413-416

25. Miyake M, Tateishi U, Maeda T, Arai Y, Hasegawa T, Sugimura K (2005) MR features of angiosarcoma in a patient with Maffucci's syndrome. Radiat Med 23:508-512

26. Vonderheide RH, Fleming MD, Carter G, Singer S, Demetri G (1997) Angiosarcoma. J Clin Oncol 15: 1708-1709

27. Kajihara M, Sugawara Y, Hirata M et al (2005) Extraskeletal osteosarcoma in the thigh: a case report. Radiat Med 23:142-146

28. Dubec JJ, Munk PL, O'Connell JX et al (1997) Soft tissue osteosarcoma with telangiectatic features: MR imaging findings in two cases. Skeletal Radiol 26:732-736

29. Murphey MD, Gibson MS, Jennings BT, Crespo-Rodriguez AM, FanburgSmith J, Gajewski DA (2006) From the archives of the AFIP: Imaging of synovial sarcoma with radiologicpathologic correlation. Radiographics 26:1543-1565

30. Tateishi U, Hasegawa T, Beppu Y, Satake M, Moriyama N (2004) Synovial sarcoma of the soft tissues: prognostic significance of imaging features. J Comput Assist Tomogr 28:140-148

31. Waldt S, Rechl H, Rummeny EJ, Woertler K (2003) Imaging of benign and malignant soft tissue masses of the foot. Eur Radiol 13:1125-1136

32. Nakanishi H, Araki N, Sawai Y et al (2003) Cystic synovial sarcomas: imaging features with clinical and histopathologic correlation. Skeletal Radiol 32:701-707

33. Stacy GS, Nair L (2007) Magnetic resonance imaging features of extremity sarcomas of uncertain differentiation. Clin Radiol 62:950-958
34. De Beuckeleer LH, De Schepper AM, Vandevenne JE et al (2000) MR imaging of clear cell sarcoma (malignant melanoma of the soft parts): a multicenter correlative MRI-pathology study of 21 cases and literature review. Skeletal Radiol 29:187-195

35. Hourani M, Khoury N, Mourany B, Shabb NS (2005) MR appearance of clear cell sarcoma of tendons and aponeuroses (malignant melanoma of soft parts): radiologic-pathologic correlation. Skeletal Radiol 34:543-546

36. Malchau SS, Hayden J, Hornicek F, Mankin HJ (2007) Clear cell sarcoma of soft tissues. J Surg Oncol 95:519-522

37. Chao KC, Chen C, Hsieh SC, Fang CL, Lao WT, Chan WP (2005) MRI of epithelioid sarcoma of the thigh. Clin Imaging 29:60-63

38. Zevallos-Giampietri EA, Barrionuevo C (2005) Proximal-type epithelioid sarcoma: report of two cases in the perineum: differential diagnosis and review of soft tissue tumors with epithelioid and/or rhabdoid features. Appl Immunohistochem Mol Morphol 13:221-230

39. Suh JS, Cho J, Lee SH et al (2000) Alveolar soft part sarcoma: MR and angiographic findings. Skeletal Radiol 29:680-689

40. Pang LM, Roebuck DJ, Griffith JF, Kumta SM, Metreweli C (2001) Alveolar soft-part sarcoma: a rare soft-tissue malignancy with distinctive clinical and radiological features. Pediatr Radiol 31:196-199

41. Vilanova JC, Woertler K, Narvaez JA et al (2007) Soft-tissue tumors update: MR imaging features according to the WHO classification. Eur Radiol $17: 125-138$ 